\title{
Comportamento Mecânico da Liga de Alumínio 2024 Submetida a Diferentes Tempos de Envelhecimento
}

\author{
F. F. CAVALCANTE ${ }^{*}$ e W. S. da SILVA ${ }^{2}$ \\ ${ }^{1}$ Instituto Federal do Rio Grande do Norte, ${ }^{2}$ Universidade Federal de Santa Catarina \\ felipe.cavalcante@ifrn.edu.br
}

Artigo submetido em 09/10/2016 e aceito em 11/11/2016

DOI: $10.15628 /$ holos.2016.5182

\section{RESUMO}

Ligas de alumínio tratáveis termicamente podem sofrer significativas alterações em suas propriedades mecânicas dependendo do tratamento realizado. Estudos recentes apontam que algumas ligas de alumínio subenvelhecidas podem experimentar o fenômeno do autorreparo ou autocura (self healing). Ou seja, quando solicitados mecanicamente, podem apresentar o fechamento ou a redução na velocidade de crescimento de trincas devido à introdução de tensões compressivas locais associadas à precipitação dinâmica de fases intermetálicas. Isso abre um leque de condições que podem ampliar novos conceitos de projetos de engenharia relacionados aos materiais metálicos principalmente no que se aplica à indústria aeronáutica. O objetivo deste trabalho é avaliar a relação entre as propriedades mecânicas e os tratamentos térmicos de uma liga de alumínio 2024 quando submetida a solubilização $\left(480^{\circ} \mathrm{C}\right.$ e $\left.2,5 \mathrm{~h}\right)$ e envelhecimento artificial $\left(190^{\circ} \mathrm{C}\right.$ por $1 \mathrm{~h}, 3 \mathrm{~h}, 5 \mathrm{~h}, 8 \mathrm{~h}$ e $\left.12 \mathrm{~h}\right)$, obtendo amostras em condições de subenvelhecimento, máximo de resistência e superenvelhecimento. Esses materiais foram caracterizados mecanicamente em relação ao ensaio de tração uniaxial (ASTM E8/E8M); do ponto de vista estrutural, através das microscopias óptica (MO), eletrônica de varredura (MEV) e microanálise por espectroscopia de energia dispersiva (EDS). Nos resultados da liga 2024 subenvelhecida esse fenômeno de autocura tornou-se palpável, pois verificou-se a ocorrência de envelhecimento dinâmico nas amostras no estado de entrega e subenvelhecidas associado a pequenos serrilhados nas curvas tensão $\mathrm{x}$ deformação. Isso traz uma excelente perspectiva para a investigação do fenômeno do autorreparo nas condições de subenvelhecimento, proporcionando o emprego de novos projetos mecânicos.

PALAVRAS-CHAVE: liga de alumínio 2024, autorreparo, precipitação dinâmica, comportamento mecânico.

\section{Mechanical Behavior of 2024 Aluminum Alloy Submitted for Different Aging Times}

\begin{abstract}
Heat treatable aluminum alloys may experience significant changes in mechanical properties depending on heat treatment developed. Recently, some researches showed that some aluminum alloys in underaged conditions may indicate the self-healing phenomenon, in other words, when stretched mechanically, the material could present a closure, or a reduction in crack growth rate due local compressive stress associated to dynamic precipitation. In this context, its valued the relationship between mechanical properties and heat treatment in 2024 aluminum alloy when submitted to solubilization $\left(480^{\circ} \mathrm{C}\right.$ for $\left.2,5 \mathrm{~h}\right)$ and artificial aging $\left(190^{\circ} \mathrm{C}\right.$ for $1 \mathrm{~h}, 3 \mathrm{~h}, 5 \mathrm{~h}, 8 \mathrm{~h}$ and $\left.12 \mathrm{~h}\right)$,
\end{abstract}

obtaining underaged, peak hardness and overaged conditions. The samples were mechanically featured using a tensile test (ASTM E8/E8M); in structural aspects, using optical microscopy, scanning electron microscopy (SEM) and energy-dispersive $\mathrm{X}$-ray spectroscopy (EDS). In underaged 2024 aluminum alloy, the phenomenon of self-healing has become tangible, because occurred dynamic precipitation on samples as received and underaged conditions, associated with small peaks in stress vs strain curves. This opens a great perspective for this investigation of self-healing in underaged conditions, providing new mechanical designs for this type of phenomenon.

KEYWORDS: 2024 aluminum alloy, aging treatments, dynamic precipitation, mechanical behavior. 


\section{INTRODUÇÃO}

Durante as últimas décadas, muita atenção foi direcionada para a avaliação da tolerância ao dano na fuselagem de aviões e sua estrutura externa (Alexopoulos, Velonaki, Stergiou \& Kourkoulis, 2016). Esse avanço no desenvolvimento das ligas de alumínio para aplicações aeronáuticas requer altos valores de tenacidade à fratura, alta performance em fadiga, grande conformabilidade, aliados ao baixo peso estrutural, grande tolerância ao dano e à alta durabilidade (Nakai \& Eto, 2000). Essas propriedades são fortemente dependentes do processo termomecânico, com isso sendo importante compreender a relação entre a evolução da microestrutura durante esses tipos de processos junto com seu comportamento mecânico (Kaçar \& Güleryüz, 2015).

A tecnologia mundial desenvolveu, até os dias de hoje, quase um milhar de ligas de alumínio, muitas das quais permanecem em uso por causa das suas características excepcionais (Carvalho, 1999). Algumas crescem de importância devido às pequenas alterações na composição ou pela adição de novos elementos, como modernas ligas desenvolvidas pela indústria aeronáutica (ligas quaternárias Al-Zn-Mg-Cu da série 7XXX), que foram desenvolvidas em grande escala para o uso em aplicações industriais (Warner, 2006).

Após Alfred Wilm, em 1906, ter descoberto o endurecimento por precipitação em uma liga de alumínio Al-Cu-Mg-Mn, foi marcado o início significativo do desenvolvimento de ligas de alumínio aeronáuticas (Liu \& Kulak, 2000). Atualmente, vários processos termomecânicos diferentes, associados ao endurecimento por envelhecimento, são bastante difundidos para os mais variados tipos de ligas de alumínio, abrindo um leque de opções para os novos projetos de engenharia. Uma possibilidade de emprego avançado dessas ligas está relacionada ao uso do fenômeno do autorreparo ou autocura (do inglês "self healing"), a partir do qual pode ser possível "fechar" ou reduzir a velocidade de crescimento de trincas, em ligas de alumínio endurecíveis por precipitação, mesmo quando estão em serviço, conforme excelentes resultados apresentados por Lumley, O’Donnell, Polmear, e Griffiths (2005), Lumley e Polmear (2007), Riemelmoser e Pippan (1998) e Zwaag (2008).

O fenômeno do autorreparo é característica essencial de biomateriais. Um exemplo típico e rotineiro seria a recuperação de fraturas ósseas ou ferimentos da pele humana. Já em materiais metálicos, devido à natureza das ligações atômicas, este fenômeno é intrinsecamente mais difícil do que em outras classes de materiais (poliméricos e cerâmicos).

Neste sentido, a proposta do autorreparo conforme descrito nas citações anteriores, insere-se no paradigma de projetos de materiais autorregeneráveis, que consistem em permitir que estes sofram transformações microestruturais controladas em resposta às condições de serviço, num processo autônomo e intrínseco, que pode estar presente, por exemplo, em ligas de alumínio de uso aeronáutico e aeroespacial. O processo é autônomo, pois não exige gatilho externo, e a energia associada ao processo que leva ao próprio dano estimula a ação de regeneração, como no caso da fadiga, no qual o encruamento decorrente da zona de deformação plástica à frente da ponta da trinca induz a precipitação de intermetálicos. Além disso, é intrínseco por não requerer agente externo de cura, uma vez que a supersaturação de soluto em liga subenvelhecida possibilitaria a elevada precipitação dinâmica de intermetálicos associada à elevada densidade de discordâncias presentes em bandas de deslizamento que ocorrem na zona de processo de crescimento da trinca de fadiga. 
Conforme discutido acima, e de forma resumida, o processo de autorreparo nas ligas de alumínio está associado à precipitação dinâmica de fases intermetálicas, que ocorre quando a liga, em condições subenvelhecidas, tende, sob a ação de carregamentos mecânicos, reparar um defeito (Lumley, Morton \& Polmear, 2002). A necessidade de condições subenvelhecidas cria uma outra questão importante a ser correlacionada: a resistência à corrosão sob tensão. De acordo com Koch e Kolijn (1979), para se estabelecer um ciclo térmico ótimo para as ligas de alumínio, deve-se levar em consideração a possibilidade de obtenção de uma ótima combinação de propriedades de resistência, como o limite de escoamento e o limite de resistência à tração, e de propriedades associadas à tolerância de trincas, como a tenacidade à fratura e a resistência à corrosão sob tensão.

\subsection{Liga 2024}

Estruturas de aviões, especialmente a asa e a fuselagem, utilizam a liga 2024 como material base devido à sua alta resistência mecânica, boa resistência à corrosão e alta capacidade de suportar danos (Alexopoulos et al., 2016; Lin, Xia, Jiang, Zhou, \& Li, 2013). Essa liga está inserida na classificação das ligas Al-Cu-Mg, cujo principal motivo de se adicionar cobre a uma liga de alumínio é aumentar a sua resistência.

A medida que se aumenta o teor de cobre, ocorre um aumento contínuo na dureza devido a formação de precipitados que consistem em um aglomerado de átomos de soluto e solvente. As zonas de Guinier-Preston, que são aglomerações com alta concentração de soluto, mantêm uma coerência com a matriz e constituem-se de obstáculos ao movimento das discordâncias de forma mais eficaz do que átomos do soluto na sua forma individual, mesmo quando a energia de interação com discordâncias associada aos átomos na solução sólida for elevada, como exemplo, em situações onde há grandes diferenças entre os diâmetros atômicos do soluto e solvente (Hirth, 1996). Essas zonas GP também se formam no envelhecimento artificial, sendo considerado precursor dos precipitados intermediários metaestáveis $\theta^{\prime \prime}$ e $\theta^{\prime}$ ou $\mathrm{S}^{\prime}$ e $S$, dependendo da composição da liga. O precipitado $\theta^{\prime \prime}$, que se forma entre três a quatro horas a $190{ }^{\circ} \mathrm{C}$ quando as zonas GP desaparecem, é coerente com a matriz, possuindo forma de plaqueta, assim como o precipitado $\theta^{\prime}$, que se forma algum tempo depois, mas coexiste com o precipitado $\theta^{\prime \prime}$, correspondendo ao máximo de dureza obtido para as ligas Al-Cu-Mg. A continuação do tratamento térmico leva a formação do precipitado de equilíbrio termodinâmico $\theta$ possuindo composição química $\mathrm{Al}_{2} \mathrm{Cu}$, em que, mesmo que o tratamento continue, não há mudança nas suas características, com exceção ao tamanho, que tende a crescer, diminuindo a dureza (superenvelhecimento) quando comparado com o intervalo de coexistência das fases $\theta^{\prime \prime}$ e $\theta^{\prime}$. O equilíbrio e o equilíbrio metaestável dessa liga a partir da condição de solução sólida supersaturada (SSSS), é dado por (Totten \& Mackenzie, 2003):

SSSS -> estado de pré-precipitação (zonas GP) $->\theta^{\prime \prime}+\theta^{\prime}->\theta\left(\mathrm{Al}_{2} \mathrm{Cu}\right)$

Lin, Xia, Jiang, \& Li (2012) mostraram que quando a razão $\mathrm{Cu} / \mathrm{Mg}$ está entre 8 e 4, os precipitados principais são $\theta\left(A \mathrm{l}_{2} \mathrm{Cu}\right)$ e $\mathrm{S}\left(A \mathrm{I}_{2} \mathrm{CuMg}\right)$. Quando esta relação está entre 4 e 1,5 , 0 principal precipitado é a fase $\mathrm{S}\left(A \mathrm{I}_{2} \mathrm{CuMg}\right)$, promovendo outra sequência de precipitação:

$$
\text { SSSS }->\mathrm{GPB}->\mathrm{S}^{\prime \prime}+\mathrm{S}^{\prime} \rightarrow \mathrm{S}\left(A \mathrm{I}_{2} \mathrm{CuMg}\right)
$$

Zonas GPB (Guinier-Preston-Bagaryatsky) são consideradas pequenas ordenações de átomos de soluto de cobre e magnésio. Ringer, Hono, Polmear, e Sakurai (1996) confirmaram que 
as zonas GPB e outras estruturas antecessoras da fase $S$ são as responsáveis pelo endurecimento por envelhecimento, enquanto que a fase $S$ aparece na condição de superenvelhecimento. $A$ formação da fase $\mathrm{S}\left(\mathrm{Al}_{2} \mathrm{CuMg}\right)$ nas ligas de $\mathrm{Al}-\mathrm{Cu}-\mathrm{Mg}$ durante o tratamento de envelhecimento é mais útil no aumento de dureza e resistência do que os precipitados $\theta\left(\mathrm{Al}_{2} \mathrm{Cu}\right)$ (Wang \& Starink, 2013).

\subsection{Tratamentos térmicos}

Tratamento térmico é, no seu sentido mais amplo, qualquer operação de aquecimento ou resfriamento realizada para modificar as propriedades mecânicas, estrutura metalúrgica ou estado de tensões internas de um produto metálico. Nas ligas de alumínio, o tratamento térmico é restrito a operações específicas utilizadas para aumentar a resistência e dureza de ligas endurecíveis por precipitação (conformadas ou fundidas).

Duas características obrigatórias devem ser exibidas pelos diagramas de fases dos sistemas das ligas para haver endurecimento por precipitação: i) deve haver uma solubilidade máxima apreciável de um componente do outro, na ordem de vários pontos percentuais; ii) deve haver um limite de solubilidade que diminua rapidamente com a concentração do componente principal com uma redução na temperatura (Mukhopadhyay, 2009).

A solubilização das ligas de alumínio visa dissolver totalmente as fases microscópicas simples ou intermediárias presentes na matriz de uma liga pelo aquecimento ao campo monofásico inerente. Logo após, pelo esfriamento mais ou menos rápido, mantém-se o estado monofásico a temperatura ambiente, tornando a liga, as vezes, mais mole e mais deformável. A solubilização é uma etapa preparatória para o futuro tratamento de endurecimento por precipitação submicroscópica, ou, envelhecimento artificial (de Azevedo Coutinho, 1980).

O tratamento térmico de precipitação ou envelhecimento tem por objetivo produzir uma fina dispersão de precipitados endurecedores submicroscópicos na matriz a partir da solução sólida supersaturada, por mecanismos de difusão auxiliados pela alta concentração de lacunas resultantes do tratamento de solubilização. Tal tratamento pode ser natural ou artificial (Fransson, 2009; Hunsicker, 1984).

Neste contexto, a ideia original deste trabalho surgiu das discussões entre professores do DEMat-CT/UFRN que têm tido trabalhos junto à indústria aeronáutica relacionados a compósitos, que indicaram esta demanda para o estudo de eventuais aprimoramentos no comportamento mecânico de ligas de alumínio para aplicações aeronáuticas (resistência à tração, tenacidade à fratura e resistência à fadiga). Daí surgiu o interesse pelo estudo do fenômeno do self-healing, que pode ter grande influência no projeto de partes estruturais para a indústria aeronáutica, uma vez que esta demanda componentes para os quais a confiabilidade é um item crucial. Com isso, esse trabalho visa contribuir através da análise de comportamento mecânico da liga 2024, especialmente no que se refere ao ensaio de tração quando a liga é submetida a diferentes tempos de envelhecimento.

\section{METODOLOGIA}

A liga 2024 foi entregue com um tratamento de solubilização, trabalho a frio e envelhecimento natural (2024-T351), possuindo dimensões $500 \times 500 \times 38$ mm, em forma de placa. 
Foi realizada uma análise de fluorescência de raios $X$ na condição de entrega de ambas as ligas, utilizando um equipamento da marca Shimadzu, modelo XRF-1800 Sequencial, com metodologia de dispersão por comprimento de onda (WD-XRF), sendo sua faixa de detecção do sódio ( $\mathrm{Na}-\mathrm{Z}=11)$ ao urânio $(U-Z=92)$ e radiação $\mathrm{RhK} \alpha(\lambda=0,615 \AA)$, no modo semi-quantitativo. A composição química é dada pela Tabela 1. Para as imagens em microscopia eletrônica, foi utilizado um MEV de bancada marca Hitachi, modelo TM-3000, interação por elétrons retroespalhados e voltagem $15 \mathrm{kV}$.

Tabela 1: Composição química das ligas após análise por fluorescência de raios $\mathrm{X}$.

\begin{tabular}{|c|c|c|c|c|c|c|c|c|}
\hline $\mathrm{Si}$ & $\mathrm{Fe}$ & $\mathrm{Cu}$ & $\mathrm{Mn}$ & $\mathrm{Mg}$ & $\mathrm{Cr}$ & $\mathrm{Zn}$ & $\mathrm{Ti}$ & $\mathrm{Al}$ \\
\hline $\begin{array}{r}0,16 \\
\pm 0,01\end{array}$ & $\begin{array}{c}0,13 \\
\pm 0,01\end{array}$ & $\begin{array}{r}4,83 \\
\pm 0,06\end{array}$ & $\begin{array}{c}0,72 \\
\pm 0,02\end{array}$ & $\begin{array}{c}1,77 \\
\pm 0,10\end{array}$ & $\begin{array}{r}0,03 \\
\pm 0,01\end{array}$ & $\begin{array}{c}0,14 \\
\pm 0,01\end{array}$ & $\begin{array}{r}0,05 \\
\pm 0,01\end{array}$ & restante \\
\hline
\end{tabular}

A caracterização microestrutural seguiu a metodologia convencional de lixamento (lixas de \#80 a \#2200 meshes), com subsequente polimento com uma politriz utilizando alumina ( $6 \mu \mathrm{m}$, $3 \mu \mathrm{m}$ e $1 \mu \mathrm{m}$ ) como abrasivo. O reagente que melhor revelou a estrutura dos grãos para as duas ligas por imersão foi o Keller $\left(100 \mathrm{ml} \mathrm{HNO}_{3}+50 \mathrm{ml} \mathrm{HCl}+1,5 \mathrm{ml} \mathrm{HF}\right)$, com tempo de imersão de 25 a 30 segundos ao invés do Poulton modificado $\left(40 \mathrm{ml} \mathrm{HNO}_{3}+30 \mathrm{ml} \mathrm{HCl}+2,5 \mathrm{ml} \mathrm{HF}+42,5 \mathrm{ml} \mathrm{H} \mathrm{H}_{2} \mathrm{O}\right.$, com tempo de imersão de 15 a 20 segundos, sendo o último melhor revelador dos precipitados, contrariando a literatura (Vander Voort, 1984). Com isso, utilizou-se Keller para a verificação do estado de entrega (distribuição dos grãos) e Poulton modificado para verificar a distribuição dos precipitados nas ligas pós tratamentos térmicos.

Para a retirada das porções de material que vão dar origem aos corpos de prova, foi utilizada uma serra circular elétrica manual, potência de $1600 \mathrm{~W}$ e rotação de $5000 \mathrm{rpm}$, com um disco dentado específico para corte em materiais metálicos. Podemos considerar que este é um método inovador e de baixo custo, visto que para grandes chapas, geralmente o corte é feito a laser (o que afeta termicamente a liga), ou utilizando uma serra fita vertical (custo elevado e de difícil disponibilidade), além de que o disco é utilizado somente para cortes em chapas de pequeno comprimento. O disco é específico para corte à seco e sem grande aumento de temperatura, o que é desejoso para o trabalho já que não promove nenhum evento térmico no material que possa comprometer alguma propriedade antes dos ensaios. Foi verificado (manualmente) que não houve nenhum aumento de temperatura significativo nas chapas e nos tarugos após o corte.

Os corpos de prova confeccionados para o ensaio de tração foram usinados com um torno CNC, com $6 \mathrm{~mm}$ de diâmetro e com comprimento útil de $60 \mathrm{~mm}$ conforme especifica a ASTM E8/E8M. O eixo de simetria das amostras possui a mesma direção de laminação, ou seja, o plano da trinca avança perpendicularmente à direção de laminação, como indica a Figura 1. O ensaio de tração se deu utilizando a máquina de ensaios universal Shimadzu, com célula de carga $300 \mathrm{kN}$ e velocidade $2 \mathrm{~mm} / \mathrm{min}$. 


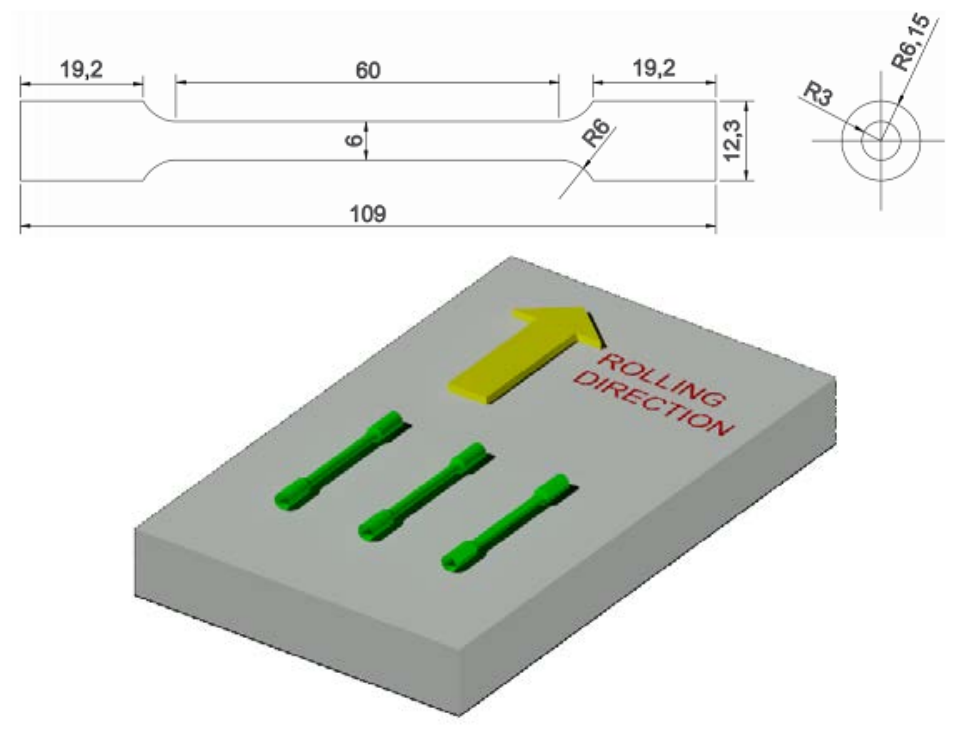

Figura 1: Dimensões do corpo de prova de tração $(6 \mathrm{~mm}$ de diâmetro e $60 \mathrm{~mm}$ de comprimento para fixação do extensômetro) e direção de retirada dos corpos de prova em relação à direção de laminação da placa.

Ainda foram confeccionados pequenos corpos de prova retangulares com medida de aproximadamente $2 \mathrm{~cm} \times 2 \mathrm{~cm} \times 38 \mathrm{~mm}$ para a realização do ensaio de dureza Vickers, utilizando a norma ASTM E92, sendo os valores de dureza obtidos pela média de seis endentações realizadas na superfície de laminação da placa. Essa superfície foi cautelosamente lixada e polida para a realização do ensaio de dureza.

Os tratamentos térmicos foram realizados utilizando dois fornos mufla sem proteção, com o objetivo de promover a solubilização $\left(480{ }^{\circ} \mathrm{C} \pm 5{ }^{\circ} \mathrm{C}\right.$ por $\left.2,5 \mathrm{~h}\right)$ e depois o envelhecimento artificial. A medida que as amostras eram solubilizadas, foram realizadas as têmperas em água até a temperatura ambiente e depois eram colocadas no outro forno com o objetivo de se prevenir qualquer envelhecimento natural. Os tempos para os envelhecimentos artificiais foram de $1 \mathrm{~h}, 3 \mathrm{~h}, 5 \mathrm{~h}, 8 \mathrm{~h}$ e $12 \mathrm{~h}$, com temperatura de $190^{\circ} \mathrm{C}$, promovendo um tratamento de têmpera T6. Esses valores de temperatura e tempo foram determinados com base na literatura (Afzal, Shah \& Ahmad, 2013; Alexopoulos et al., 2016; Kaçar \& Güleryüz, 2015; Radutoiu, Alexis, Lacroix, Petit, Abrudeanu, Rizea \& Vulpe, 2012). Para o ensaio de tração foram produzidas doze amostras (duas para cada tempo de envelhecimento mais duas para o estado de entrega). Já para o ensaio de dureza, foram confeccionados seis corpos de prova (uma para cada tempo de envelhecimento, mais uma para o estado de entrega).

As amostras prontamente confeccionadas e tratadas termicamente foram ensaiadas no dia subsequente ao término do último tratamento térmico realizado. O objetivo é manter o mínimo de tempo entre os tratamentos e o ensaio para que nenhum efeito de precipitação natural pós tratamento possa ocorrer, já que foi comprovado por Lumley et al. (2002) que o atraso entre o tratamento e o ensaio pode mudar significativamente o efeito e as propriedades das condições subenvelhecidas das ligas de alumínio.

Valores de porcentagem de redução de área $\left(A_{\circ}-A_{f}\right) / A_{\circ}$ foram calculados medindo-se os diâmetros com um paquímetro, após a união das duas partes fraturadas, bem próximo das superfícies de ruptura onde ocorreu o fenômeno da estricção. 
Os valores de tensão de escoamento foram obtidos traçando-se uma reta paralela à fase elástica do ensaio a partir do valor de deformação de $0,2 \%$ utilizando o software Origin ${ }^{\circledR}$ para aquisição mais precisa dos dados. Já a deformação longitudinal foi calculada pela relação ( $L_{f}$ $\left.L_{0}\right) / L_{0}$ medindo-se o comprimento da área útil da amostra antes da fratura e após a fratura.

\section{RESULTADOS E DISCUSSÕES}

Para a liga 2024, os elementos principais ( $\mathrm{Cu}$ e $\mathrm{Mg}$ ) estão presentes em porcentagens próximas aos limites superiores, 4,9 \%peso e 1,8 \%peso, respectivamente, como estabelecido pelo Handbook (1979). Como os teores de cobre e magnésio são altos, ocorre um oferecimento ao endurecimento bastante elevado, conforme propõe a literatura (Sanders, Baumann \& Stumpf, 1986).

Curvas de engenharia representativas de cada condição estão apresentadas na Figura 2. As condições de estado de entrega, subenvelhecida (1h) e aquela dita como a de máxima dureza (envelhecimento por $3 \mathrm{~h}$ ) apresentam alta ductilidade e resistência mecânica um pouco inferior às ligas superenvelhecidas, em princípio, contrariando o senso comum de que maiores durezas estão associadas a maiores resistências à tração e a menores ductilidades. As ligas superenvelhecidas apresentam, por sua vez, elevação na resistência mecânica e redução na ductilidade. Esta mudança pode estar associada a duas questões: i) de um lado a maior fração volumétrica de precipitados nestas ligas reduz a mobilidade de discordâncias, elevando a resistência do material; ii) do outro, a presença de precipitados em tamanhos maiores facilita a nucleação de micro vazios e, por consequência, a nucleação de trincas, comprometendo com isso a ductilidade do material.

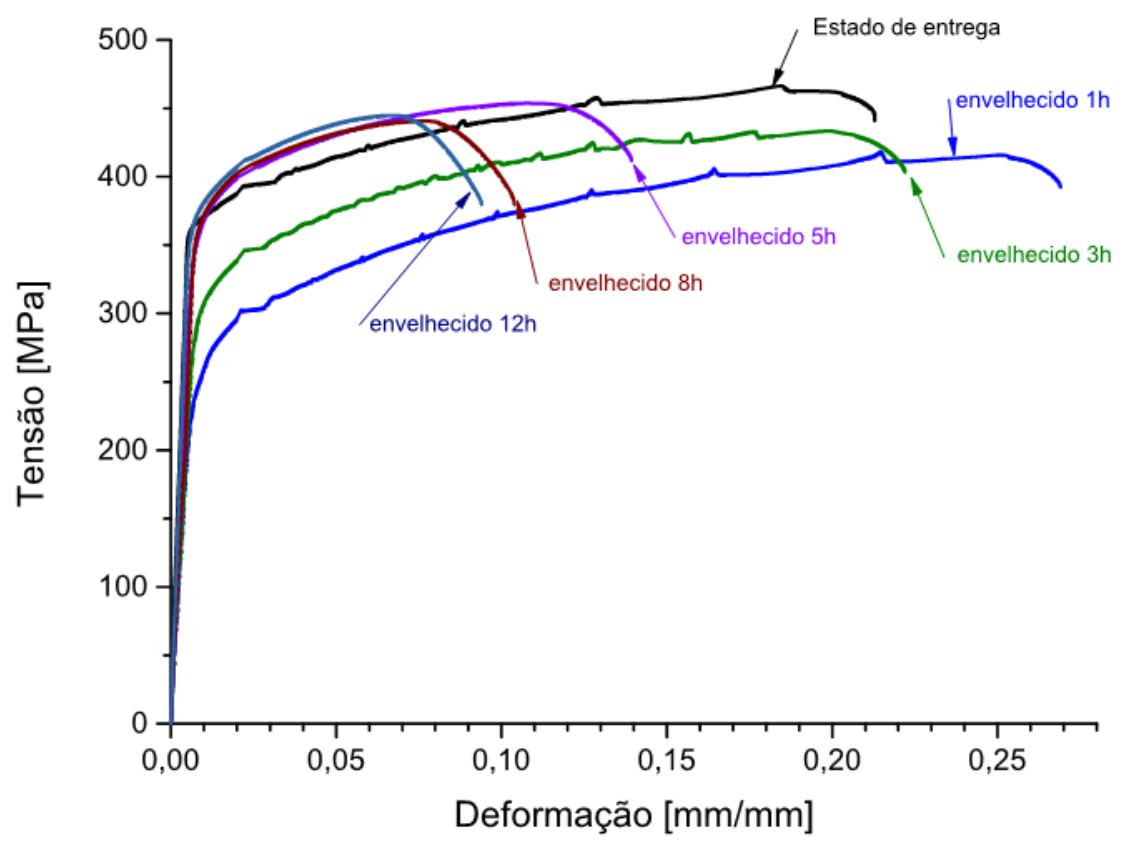

Figura 2: Curvas características obtidas nos ensaios de tração para a liga 2024 em diferentes tempos de envelhecimento.

Os pequenos picos dos serrilhados (aumento nos valores de tensão) apresentados pelo gráfico, acredita-se que são consequências dos impedimentos dos movimentos das discordâncias 
devido à precipitação (neste caso, dinâmica), que ocorreram exatamente nas condições que ainda apresentam grandes quantidades de átomos em solução devido aos parâmetros de tratamentos propostos, possuindo um alto potencial para a precipitação, sendo a tensão de tração aplicada, a força motriz para que essa precipitação venha a ocorrer, consequentemente, elevando os valores de tensões em pontos específicos e bastante perceptíveis. Nos dois corpos de provas ensaiados para cada condição, a curva apresentou o mesmo comportamento, ou seja, os picos estão em intervalos bem definidos para cada valor de tensão-deformação para as amostras no estado de entrega, envelhecido por 1 h e envelhecido por 3 h. Não foi encontrado na literatura nenhum comportamento parecido como apresentado por esse gráfico, levando a possibilidade de uma análise mais precisa, como um aumento na quantidade de corpos de prova para os mesmos parâmetros de tratamentos térmicos e ainda, obter menores intervalos de tempo de envelhecimento entre $1 \mathrm{~h}$ e $3 \mathrm{~h}$.

Este evento se apresenta como bastante interessante para os propósitos da linha de pesquisa, uma vez que o autorreparo esperado para algumas ligas de alumínio, depende deste fenômeno de envelhecimento dinâmico, conforme discutido por Lumley e colaboradores em diversas publicações (Lumley et al., 2002; Lumley, 2007; Lumley \& Polmear, 2007). A frequência do serrilhado variou para cada uma das condições de tratamento, indicando diferentes graus de saturação em solutos da matriz de alumínio, portanto diferentes potenciais de precipitação de intermetálicos endurecedores. Novamente, o melhor entendimento do fenômeno demandará novas análises, uma vez que a literatura específica sobre este fenômeno em ligas de alumínio mostrou-se escassa.

Outro fato interessante a ser notado é que, o gráfico exposto na Figura 3, que relaciona dureza Vickers $\left(\mathrm{HV}_{15}\right)$ com a porcentagem de redução de área e tempo de envelhecimento, aponta algo também não comum. Para o tempo de 3 h de envelhecimento (máxima dureza) o material nessa condição apresenta os maiores valores de redução de área $(38,26 \% \pm 0,93 \%)$ quando comparadas as outras condições.

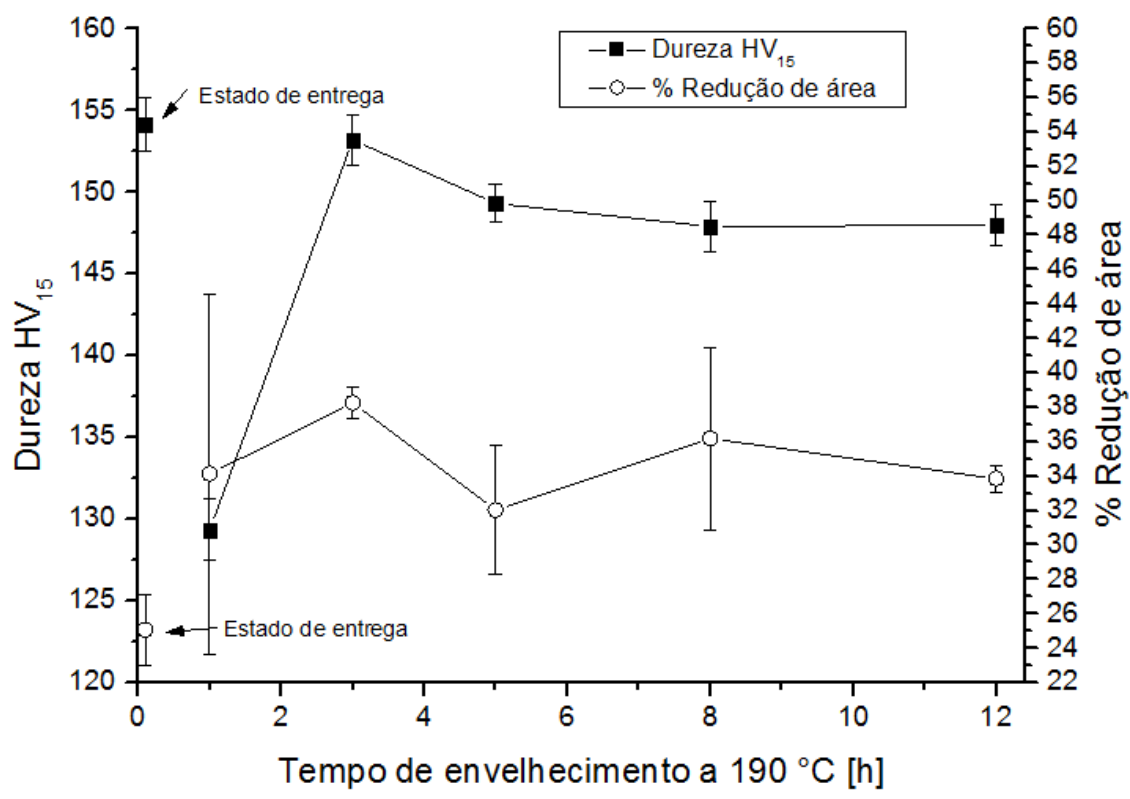

Figura 3: Relação entre dureza, redução de área e tempo de envelhecimento da liga 2024. 
A relação entre tensão máxima, tensão de escoamento e deformação longitudinal está expressa na Figura 4. Valores de tensão máxima foram obtidos pelo próprio software da máquina de ensaio (Trapezium $X^{\circledR}$ ). Pelo gráfico, pode ser observado que a tensão de escoamento tende a uma certa relação proporcional com o limite de resistência à tração. Porém, como a liga na condição de envelhecimento por $3 \mathrm{~h}$ apresenta alto valor de dureza e relativo alto valor de redução de área, e, observando o comportamento da deformação longitudinal, ocorre relação proporcional com a alta estricção, visto que os valores foram próximos na condição de mais baixa dureza (1h), confirmando que a liga para o envelhecimento de $3 \mathrm{~h}$ (máxima dureza), apresenta tanto elevado valor de redução de área, como de deformação longitudinal.

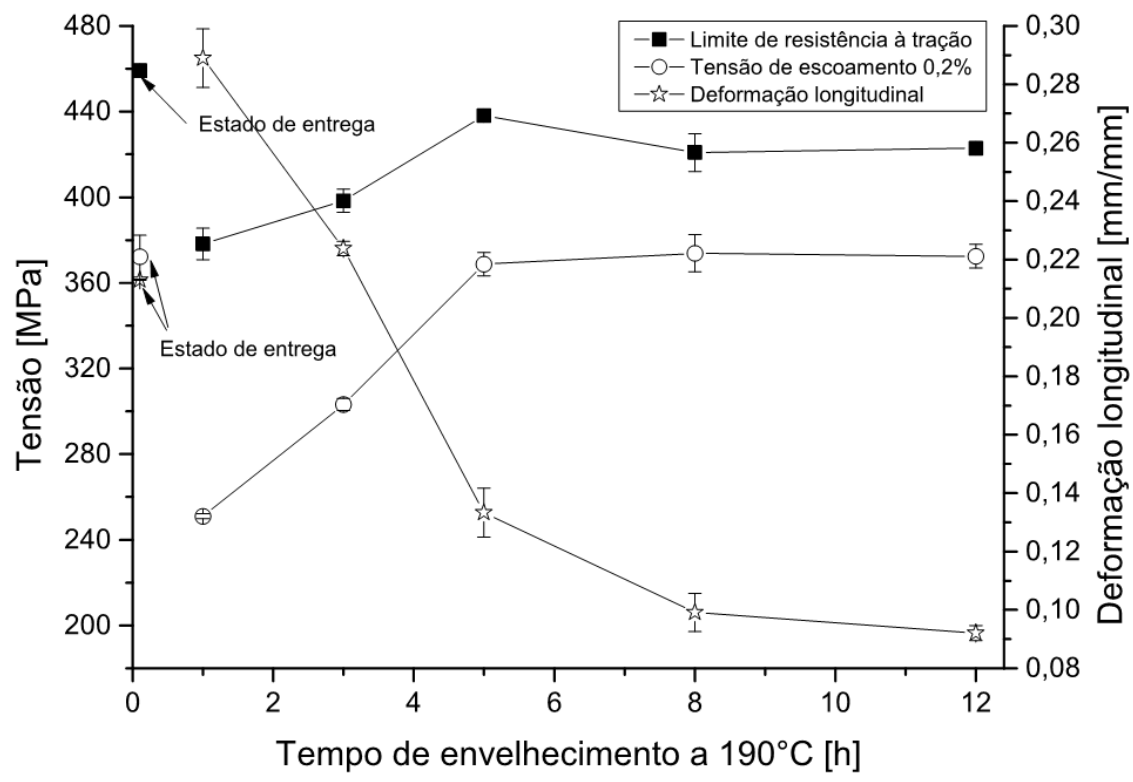

Figura 4: Relação entre limite de resistência à tração, tensão de escoamento e deformação longitudinal da liga 2024.

O gráfico exibido na Figura 5 mostra a relação dos valores de tenacidade (obtidos através do software $\operatorname{Origin}^{\circledR}$ que, por análise de dados, calculou a área abaixo das curvas de engenharia) com a deformação longitudinal. Altos valores de tenacidade são alcançados na condição de estado de entrega, subenvelhecimento (1h), inclusive máxima dureza, confirmando ainda mais a alta plasticidade ocasionada por essas condições. Tenacidade e deformação longitudinal apresentaram comportamentos bastantes similares para essas ligas. 


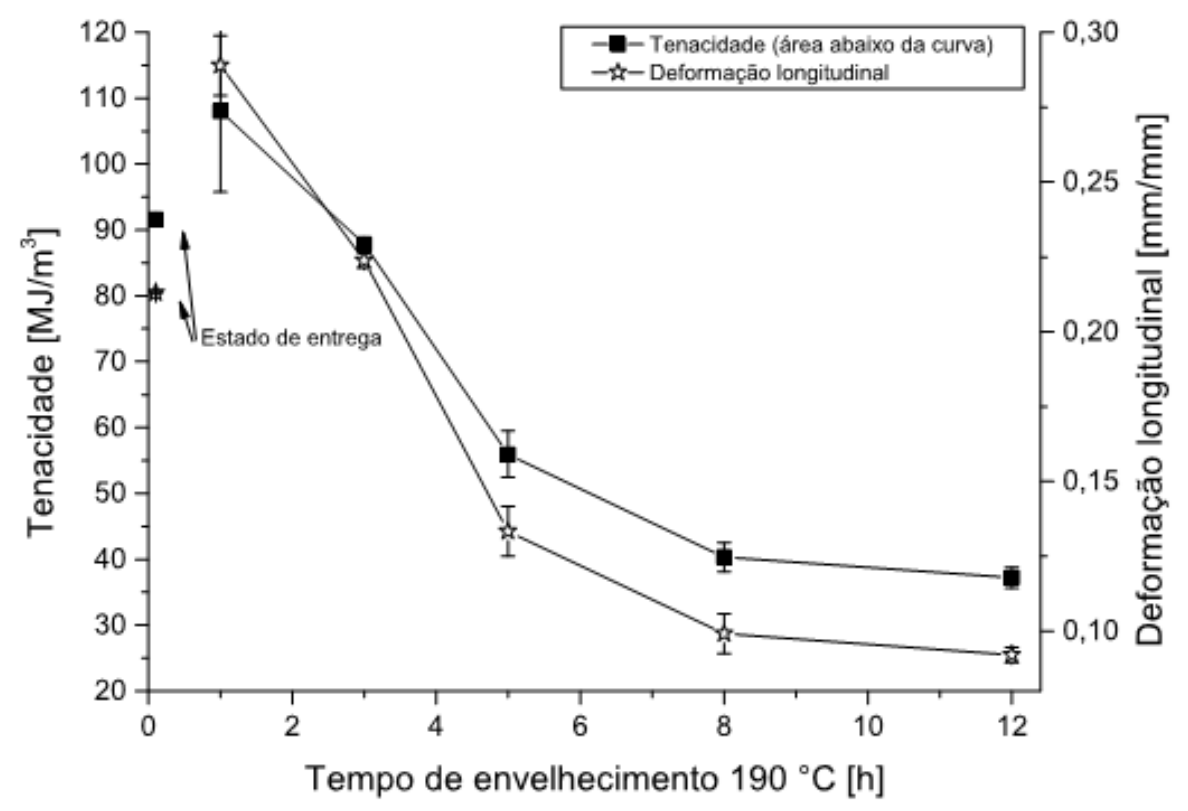

Figura 5: Relação entre tenacidade, deformação longitudinal e tempo de envelhecimento da liga 2024.

Na micrografia do estado de entrega apresentada pela Figura 6 há uma distribuição em cadeia dos precipitados insolúveis (em preto), resultados do processo de fundição ou solidificação das ligas (Lin et al., 2013), na matriz de alumínio ao longo da direção de laminação. Apesar da grande quantidade de cobre e magnésio oferecerem um grande potencial para o endurecimento, a grande quantidade de precipitados grosseiros distribuídos na matriz de alumínio fragilizarão o material, o que resultará em uma baixa resistência (Lin et al., 2013).
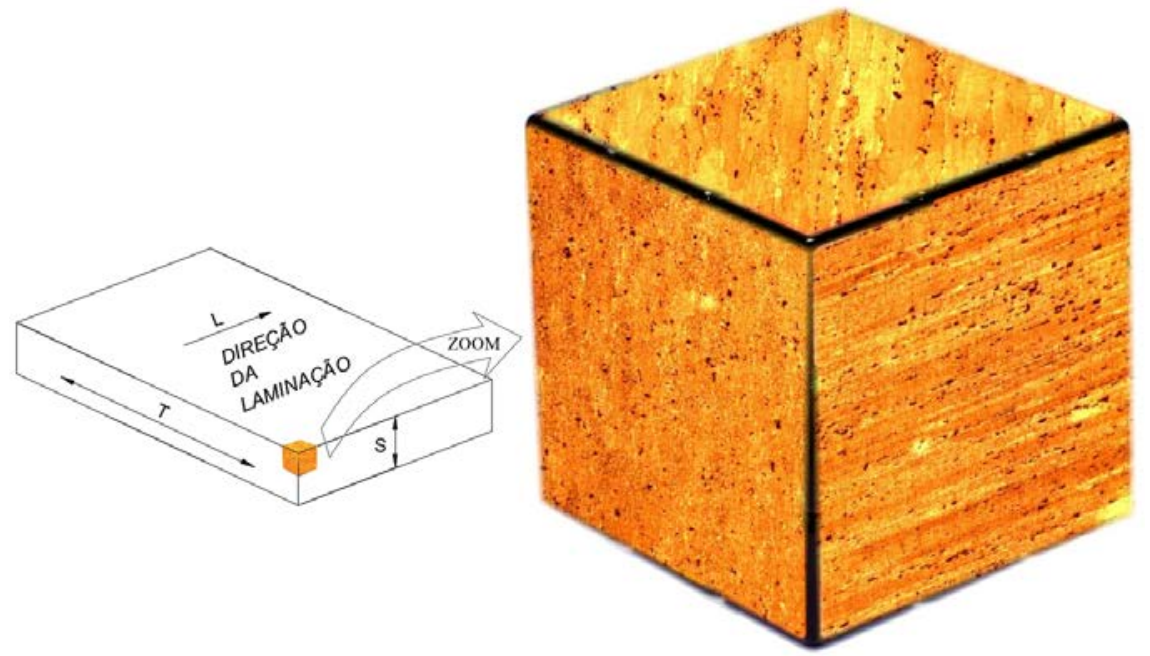

Figura 6: Direções L, T e S referente as micrografias da estrutura 3D da placa 2024. Amplificação 50x. Ataque Keller.

Tendo em vista que os precipitados que promovem as mudanças mais significativas em relação às propriedades mecânicas após os tratamentos térmicos, inclusive à autocura, são partículas submicroscópicas (não sendo caracterizadas por MEV), do ponto de vista macroscópico, as micrografias apresentam uma matriz de alumínio cinza com partículas insolúveis e grosseiras na microestrutura, que são fases de equilíbrio na liga (Figura 7). A principal razão para a formação dessas partículas (precipitados) é a quantidade de elementos adicionados na matriz de alumínio que excedem o limite de solubilidade. Embora os precipitados insolúveis 
sejam duros e frágeis, sua precipitação promove um melhor papel no endurecimento da liga se sua quantidade e distribuição tendem a uma significativa homogeneidade. Caso contrário, uma grande quantidade dessas partículas insolúveis promoverão certa fragilização no material, resultando em uma baixa resistência mecânica, pois eles são pontos de concentrações de tensões, promovendo as formações dos primeiros vazios no material.

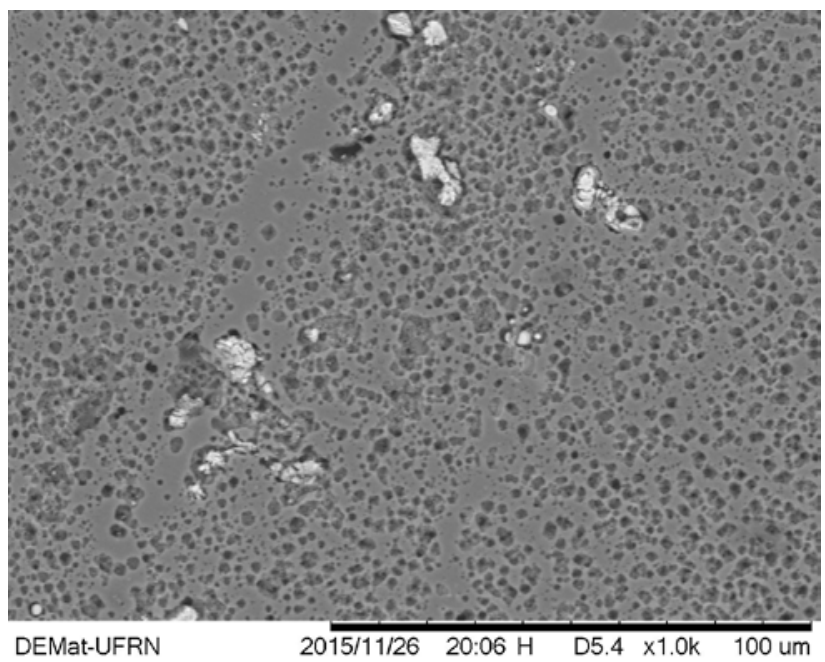

a) Estado de entrega

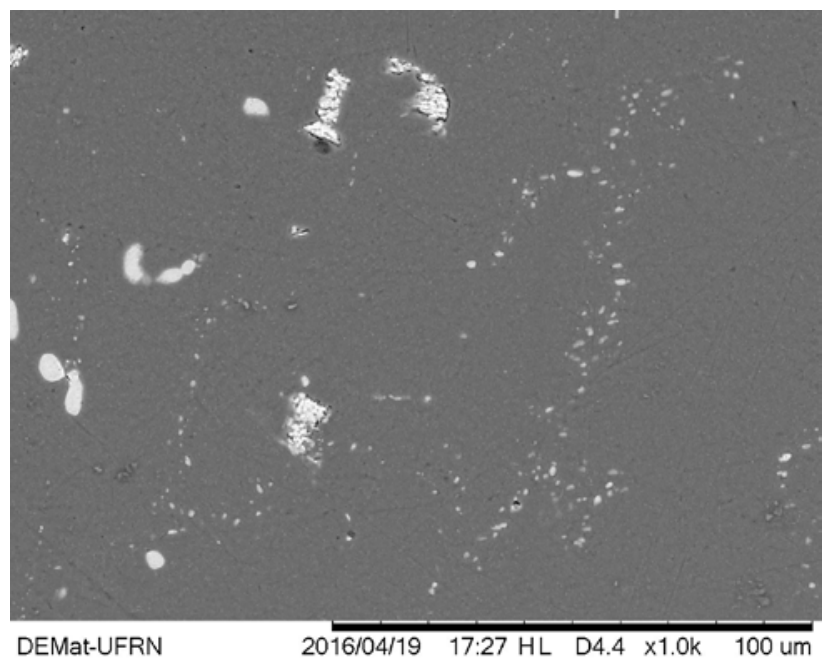

C) Solubilizada e envelhecida a $190{ }^{\circ} \mathrm{C}$ por $3 \mathrm{~h}$

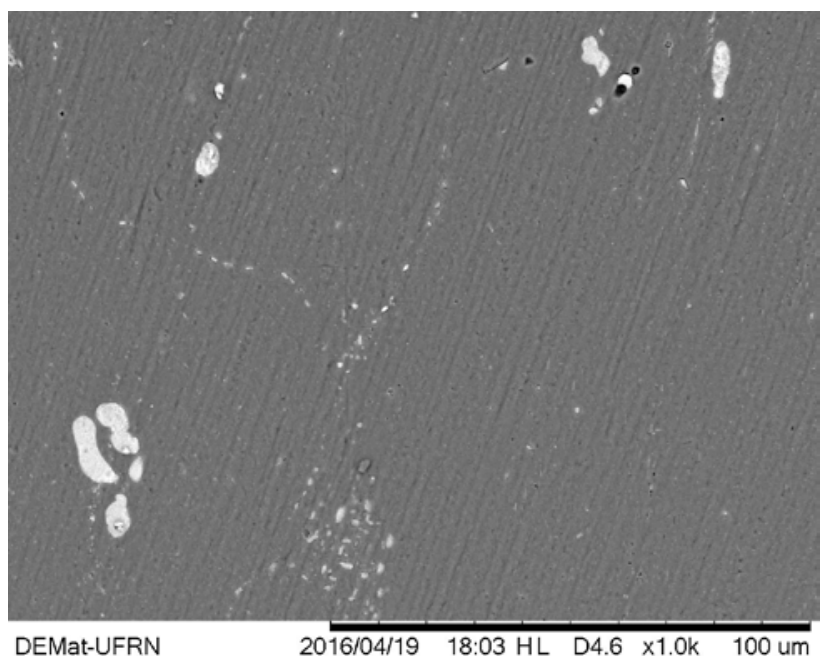

b) Solubilizada e envelhecida a $190^{\circ} \mathrm{C}$ por $1 \mathrm{~h}$

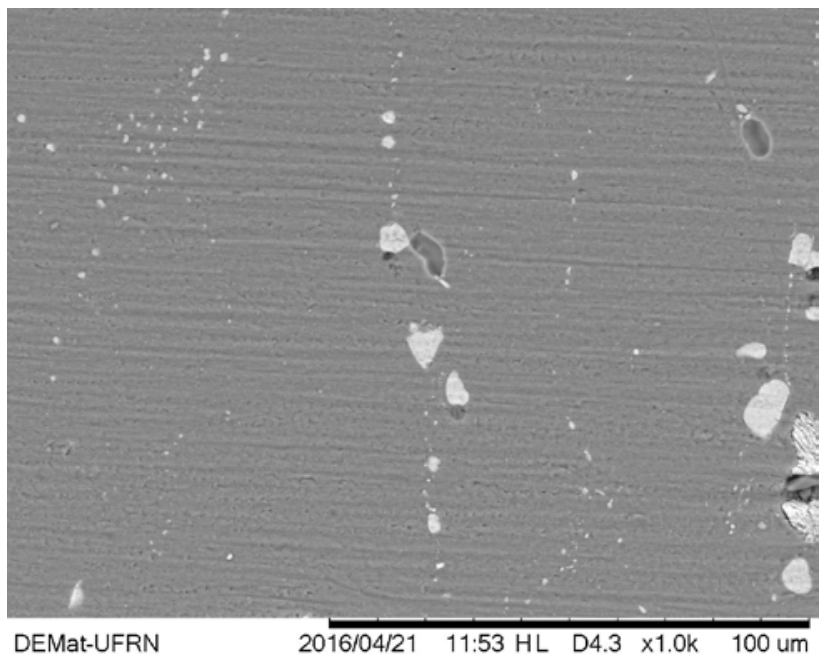

d) Solubilizada e envelhecida $190^{\circ} \mathrm{C}$ por $12 \mathrm{~h}$

Figura 7: Comparação das micrografias da liga 2024 obtidas por MEV do Estado de Entrega (a), subenvelhecida (1h) (b), máxima dureza (3h) (c) e superenvelhecida (12h) (d). Elétrons retroespalhados e ataque Poulton modificado.

A Figura 8 mostra que existem, basicamente, cinco tipos de precipitados que são bastante distinguíveis: os precipitados maiores com aparência mais "rugosa", dando a impressão de um certo relevo (PONTO 1); precipitados com aparência mais "lisa" e clara, que aparentam estar no mesmo plano da matriz de alumínio e de tamanho médio menor do que os rugosos - acredita-se que esses últimos sejam os precipitados $\mathrm{S}\left(\mathrm{Al}_{2} \mathrm{CuMg}\right)$, visto que esta composição é a que mais se aproxima estequiometricamente desse tipo de precipitado; o precipitado mais facetado em tom 
de cinza escuro (PONTO 3); o precipitado mais escuro envolto de uma região mais clara (PONTO 4); e os precipitados parecidos com os mostrados no PONTO 2, apesar de serem mais claros, apresentam certas "manchas" cinzas no plano do precipitado, sendo os únicos a possuir pouca quantidade de zinco (PONTO 5). A Tabela 2 apresenta a porcentagem atômica de cada precipitado nas micrografias mostradas nas Figuras $8 \mathrm{a}$ e $8 \mathrm{~b}$.

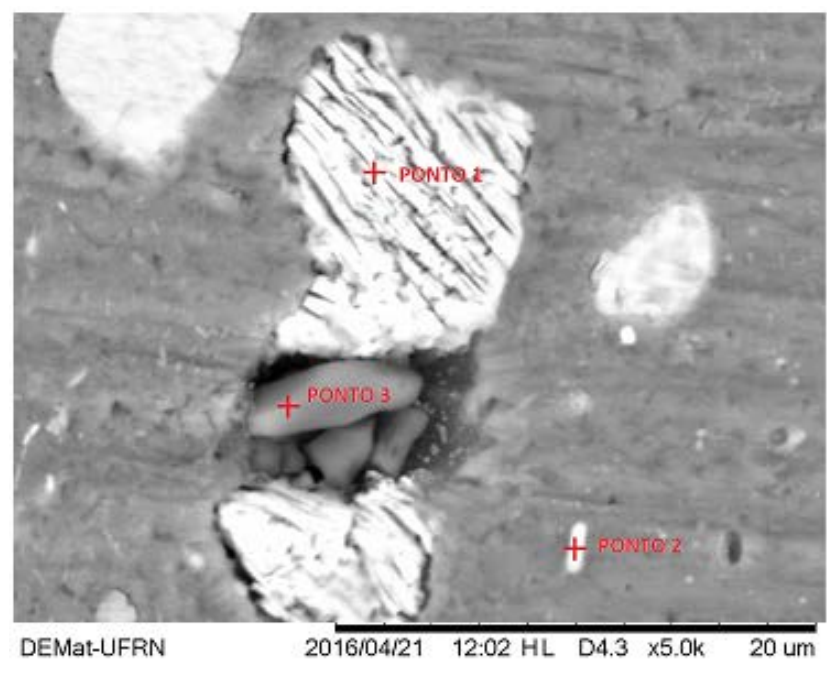

a)

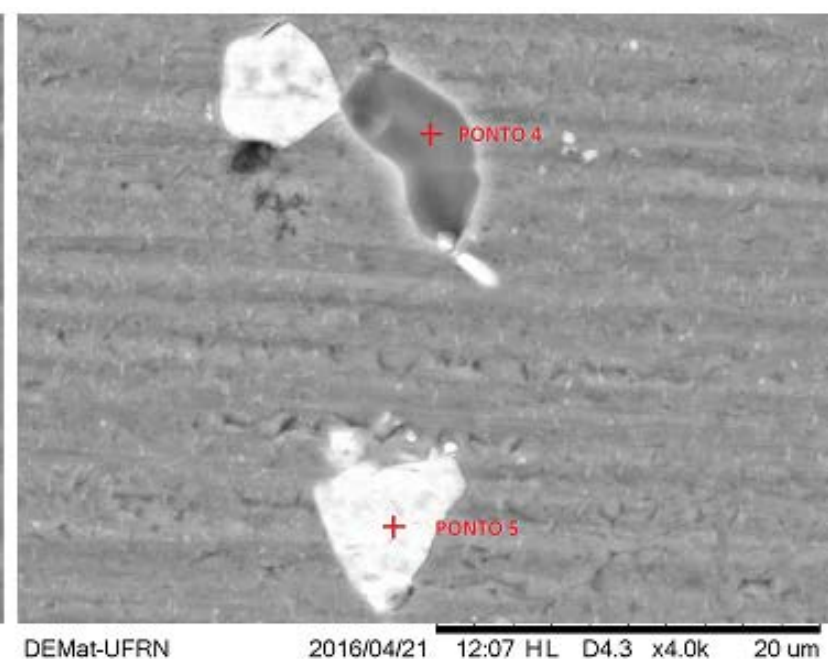

b)

Figura 8: Precipitados mais perceptíveis encontrados na liga 2024. Elétrons retroespalhados e ataque Poulton modificado.

Tabela 2: Composição química dos precipitados após microanálise por EDS.

\begin{tabular}{c|c|c|c|c|c|c|c}
\hline & \multicolumn{7}{c}{ \% atômica } \\
\hline & Al & Mg & Mn & Fe & Cu & Cr & Zn \\
\hline Ponto 1 & 41,2 & - & 0,4 & 4,7 & 53,2 & 0,5 & - \\
\hline Ponto 2 & 61,5 & 7,5 & 0,3 & 0,9 & 29,8 & - & - \\
\hline Ponto 3 & 66,3 & - & - & 8,1 & 25,7 & - & - \\
\hline Ponto 4 & 95,1 & 1,9 & 1,3 & 0,1 & 1,7 & - & - \\
\hline Ponto 5 & 70,5 & 4,4 & 0,2 & 0,5 & 22,0 & - & 2,2 \\
\hline
\end{tabular}

A Tabela 3 resume os valores de propriedades obtidas (módulo de elasticidade, E; tensão de escoamento $0,2 \%$, SY; limite de resistência à tração, UTS; porcentagem de redução de área, RA; deformação longitudinal, عf; e tenacidade, UT) no ensaio de tração para a liga 2024 nas mais variadas condições de envelhecimento. Esses dados são de extrema importância para o desenvolvimento dessa linha de pesquisa que relaciona a liga de alumínio com o fenômeno da autorreparo, tendo em vista que a literatura especializada se mostrou muito escassa quanto às condições de sub e superenvelhecimento. 
Tabela 3: Propriedades gerais obtidas no ensaio de tração da liga 2024.

\begin{tabular}{c|c|c|c|c|c|c}
\hline Condição & $\begin{array}{c}\mathrm{E} \\
{[\mathrm{GPa}]}\end{array}$ & $\begin{array}{c}\text { SY } \\
{[\mathrm{MPa}]}\end{array}$ & $\begin{array}{c}\text { UTS } \\
{[\mathrm{MPa}]}\end{array}$ & $\begin{array}{c}\mathrm{RA} \\
\%\end{array}$ & $\begin{array}{c}\varepsilon f \\
{[\mathrm{~m} / \mathrm{m}]}\end{array}$ & $\begin{array}{c}\text { UT } \\
{\left[\mathrm{MJ} / \mathrm{m}^{3}\right]}\end{array}$ \\
\hline \multirow{2}{*}{ Recebido } & 64,64 & 372,15 & 467,5 & 25,07 & 0,213 & 91,63 \\
& $\pm 1,25$ & $\pm 10,03$ & $\pm 1,73$ & $\pm 2,05$ & $\pm 0,002$ & $\pm 0,09$ \\
\hline \multirow{2}{*}{$1 \mathrm{~h}$} & 66,73 & 251,02 & 418,9 & 34,13 & 0,288 & 108,05 \\
& $\pm 19,54$ & $\pm 1,18$ & $\pm 4,41$ & $\pm 10,45$ & $\pm 0,028$ & $\pm 12,29$ \\
\hline \multirow{2}{*}{$3 \mathrm{~h}$} & 63,93 & 303,22 & 431,05 & 38,26 & 0,224 & 87,89 \\
& $\pm 6,52$ & $\pm 2,78$ & $\pm 3,26$ & $\pm 0,93$ & $\pm 0,003$ & $\pm 0,6$ \\
\hline \multirow{2}{*}{$5 \mathrm{~h}$} & 62,05 & 368,67 & 454,89 & 32,02 & 0,133 & 55,95 \\
& $\pm 3,61$ & $\pm 5,42$ & $\pm 1,68$ & $\pm 3,73$ & $\pm 0,008$ & $\pm 3,52$ \\
\hline \multirow{2}{*}{$8 \mathrm{~h}$} & 63,14 & 373,89 & 444,54 & 36,18 & 0,099 & 40,34 \\
& $\pm 5,57$ & $\pm 8,68$ & $\pm 5,32$ & $\pm 5,31$ & $\pm 0,007$ & $\pm 2,22$ \\
\hline \multirow{2}{*}{$12 \mathrm{~h}$} & 67,93 & 372,47 & 445,7 & 33,85 & 0,099 & 37,20 \\
& $\pm 0,29$ & $\pm 5,48$ & $\pm 1,17$ & $\pm 0,78$ & $\pm 0,003$ & $\pm 1,59$ \\
\hline \multicolumn{7}{|c}{}
\end{tabular}

\section{CONCLUSÕES}

Neste trabalho, o comportamento mecânico da liga 2024 submetida a diferentes tempos de envelhecimento ( $1 \mathrm{~h}, 3 \mathrm{~h}, 5 \mathrm{~h}, 8 \mathrm{~h}$ e $12 \mathrm{~h}$ ) pode ser resumido como:

- A liga 2024 apresentou um comportamento sob tração bastante dependente das condições de envelhecimento.

- As amostras subenvelhecidas apresentaram indícios do fenômeno da precipitação dinâmica e maior tenacidade, ductilidade (maior redução de área e maior deformação no instante da fratura). A identificação da ocorrência da precipitação dinâmica nessas ligas provoca boa perspectiva para a investigação do fenômeno do autorreparo nas condições de subenvelhecimento, demandando cada vez mais dados relativos a esse tipo de estudo.

- Técnicas de caracterização microestrutural utilizadas neste trabalho não permitiram estabelecer com mais profundidade características microestruturais mais peculiares e presentes nas diferentes condições de tratamentos térmicos, especialmente no caso das precipitações secundárias.

- No caso das ligas superenvelhecidas, verifica-se uma redução na ductilidade e na tenacidade, em que pese os valores mais baixos de dureza. Este fenômeno pode estar associado ao engrossamento dos precipitados com o tempo de envelhecimento, o que facilita a nucleação de defeitos e, por consequência, a nucleação de trincas, reduzindo a capacidade do material em absorver energia de deformação.

\section{REFERÊNCIAS BIBLIOGRÁFICAS}

1. Afzal, N., Shah, T., \& Ahmad, R. (2013). Microstructural Features and Mechanical Properties of Artificially Aged AA2024. Strength of Materials, 45(6), 684-692.

2. Alexopoulos, N. D., Velonaki, Z., Stergiou, C. I., \& Kourkoulis, S. K. (2016). The effect of artificial ageing heat treatments on the corrosion-induced hydrogen embrittlement of 2024 (Al-Cu) aluminium alloy. Corrosion Science, 102, 413-424. 
3. Carvalho, A. L. M. (1999). Comportamento mecânico das ligas de alumínio 7010-T74 e 7050T7451 de aplicação aeronáutica. (Dissertação Mestrado)-Faculdade de Engenharia Mecânica, Universidade Estadual Paulista Júlio de Mesquita Filho, Guaratinguetá.

4. de Azevedo Coutinho, T. (1980). Metalografia de não ferrosos: análise e prática. Edgard Blucher.

5. Fransson, C. (2009). Accelerated aging of aluminum alloys.

6. Handbook, M. (1979). Properties and selection: nonferrous alloys and pure metals. American Society for Metals, Metals Park, $\mathrm{OH}$.

7. Hirth, J. (1996). Chapter 20 - dislocations. In: Haasen, R. W. C. (Ed.). Physical Metallurgy (Fourth Edition). Fourth edition. Oxford: North-Holland (pp. 1831 - 1875). ISBN 978-0-44489875-3. Disponível em: <http://www.sciencedirect.com/science/article/pii/ B9780444898753500259>

8. Hunsicker, H. Y. (1984). Metallurgy of heat treatment and general principles of precipitation hardening. In Aluminium-Properties and Physical Metallurgy (pp. 152-157). American Society for Metals $\mathrm{OH}$.

9. Kaçar, R., \& Güleryüz, K. (2015). Effect of Quenching Rate and Pre-strain on the Strain Ageing Behaviors of 7075 Aluminum Alloys. Materials Research, 18(2), 328-333.

10. Koch, G. H., \& Kolijn, D. T. (1979). The heat treatment of the commercial aluminum alloy 7075. Journal of Heat Treating, 1(2), 3-14.

11. Lin, Y. C., Xia, Y. C., Jiang, Y. Q., \& Li, L. T. (2012). Precipitation in Al-Cu-Mg alloy during creep exposure. Materials Science and Engineering: A, 556, 796-800.

12. Lin, Y. C., Xia, Y. C., Jiang, Y. Q., Zhou, H. M., \& Li, L. T. (2013). Precipitation hardening of 2024-T3 aluminum alloy during creep aging. Materials Science and Engineering: A, 565, 420429.

13. Liu, J., \& Kulak, M. (2000). A new paradigm in the design of aluminum alloys for aerospace applications. In Materials Science Forum (Vol. 331, pp. 127-142). Trans Tech Publications.

14. Lumley, R. (2007). Self healing in aluminium alloys. In Self Healing Materials (pp. 219-254). Springer Netherlands.

15. Lumley, R. N., Morton, A. J., \& Polmear, I. J. (2002). Enhanced creep performance in an Al$\mathrm{Cu}-\mathrm{Mg}-\mathrm{Ag}$ alloy through underageing. Acta Materialia, 50(14), 3597-3608.

16. Lumley, R. N., O’Donnell, R. G., Polmear, I. J., \& Griffiths, J. R. (2005). Enhanced fatigue resistance by underageing an Al-Cu-Mg-Ag alloy. In Materials Forum (Vol. 29, pp. 256-261).

17. Lumley, R., \& Polmear, I. J. (2007). Advances in self healing of metals. Self healing materials: an alternative approach to, 20, 219-254.

18. Mukhopadhyay, A. K. (2009). Microstructure and properties of high strength aluminium alloys for structural applications. Transactions of the Indian Institute of Metals, 62(2), 113122.

19. Nakai, M., \& Eto, T. (2000). New aspect of development of high strength aluminum alloys for aerospace applications. Materials Science and Engineering: A, 285(1), 62-68.

20. Radutoiu, N., Alexis, J., Lacroix, L., Petit, J. A., Abrudeanu, M., Rizea, V., \& Vulpe, S. (2012). 
Effect of the over-ageing treatment on the mechanical properties of AA2024 aluminum alloy. Revista de chimie (chemistry magazine), 63, pp-1042.

21. Riemelmoser, F. O., \& Pippan, R. (1998). Mechanical reasons for plasticity-induced crack closure under plane strain conditions. Fatigue \& fracture of engineering materials \& structures, 21(12), 1425-1433.

22. Ringer, S. P., Hono, K., Polmear, I. J., \& Sakurai, T. (1996). Nucleation of precipitates in aged Al Cu Mg (Ag) alloys with high Cu: Mg ratios. Acta Materialia, 44(5), 1883-1898.

23. Sanders, R. E., Baumann, S. F., \& Stumpf, H. C. (1986). Non-heat-treatable aluminum alloys. Aluminum Alloys: Their Physical and Mechanical Properties., 3, 1441-1484.

24. Totten, G. E., \& Mackenzie, D. S. (Eds.). (2003). Handbook of Aluminum: Vol. 1: Physical Metallurgy and Processes (Vol. 1). CRC Press.

25. Vander Voort, G. F. (1984). Metallography, principles and practice. ASM International.

26. Wang, S. C., \& Starink, M. J. (2013). Precipitates and intermetallic phases in precipitation hardening $\mathrm{Al}-\mathrm{Cu}-\mathrm{Mg}-(\mathrm{Li})$ based alloys. International Materials Reviews.

27. Warner, T. (2006). Recently-developed aluminium solutions for aerospace applications. In Materials Science Forum (Vol. 519, pp. 1271-1278). Trans Tech Publications.

28. Zwaag, S. (Ed.). (2008). Self healing materials: an alternative approach to 20 centuries of materials science. Springer Science+ Business Media BV. 